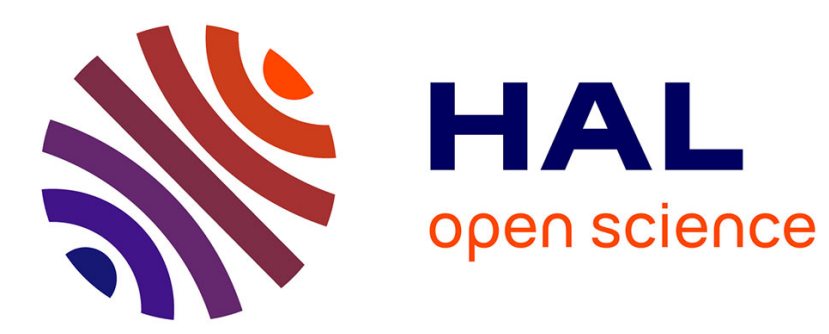

\title{
Experimental and theoretical study of the passively mode-locked Ytterbium-doped double-clad fiber laser
}

Hervé Leblond, Mohamed Salhi, Ammar Hideur, Thierry Chartier, Marc Brunel, François Sanchez

\section{- To cite this version:}

Hervé Leblond, Mohamed Salhi, Ammar Hideur, Thierry Chartier, Marc Brunel, et al.. Experimental and theoretical study of the passively mode-locked Ytterbium-doped double-clad fiber laser. Physical Review A : Atomic, molecular, and optical physics [1990-2015], 2002, 65, pp.063811. 10.1103/PhysRevA.65.063811 . hal-00003091

\section{HAL Id: hal-00003091 https://hal.science/hal-00003091}

Submitted on 18 Oct 2004

HAL is a multi-disciplinary open access archive for the deposit and dissemination of scientific research documents, whether they are published or not. The documents may come from teaching and research institutions in France or abroad, or from public or private research centers.
L'archive ouverte pluridisciplinaire HAL, est destinée au dépôt et à la diffusion de documents scientifiques de niveau recherche, publiés ou non, émanant des établissements d'enseignement et de recherche français ou étrangers, des laboratoires publics ou privés. 


\title{
Experimental and theoretical study of the passively mode-locked Ytterbium-doped double-clad fiber laser
}

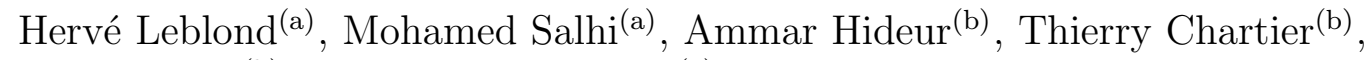

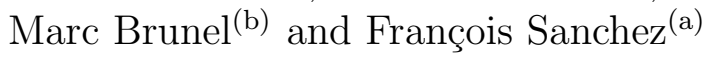

(a) Laboratoire POMA, UMR 6136, Université d'Angers, 2 Bd Lavoisier, 49000 Angers, France

(b) Groupe d'Optique et d'Optronique, CORIA, UMR 6614, Université de Rouen, Avenue de l'Université, site Universitaire du Madrillet, B.P. 12, 76801 Saint-Étienne du Rouvray, France

\begin{abstract}
We consider an Yb-doped double-clad fiber laser in a unidirectional ring cavity containing a polarizer placed between two half-wave plates. Depending on the orientation of the phase plates, the laser operates in continuous, Qswitch, mode-lock or unstable self-pulsing regime. An experimental study of the stability of the mode locking regime is realized versus the orientation of the half-wave plates. A model for the stability of self-mode-locking and cw operation is developed starting from two coupled nonlinear Schrödinger equations in a gain medium. The model is reduced to a master equation in which the coefficients are explicitly dependent on the orientation angles of the phase plates. Analytical solutions are given together with their stability versus the angles.
\end{abstract}




\section{Introduction}

Self-mode-locking has been successfully obtained in both low power and double-clad fiber lasers using Additive-Pulse Mode locking technique through nonlinear polarization rotation [1]-[6]. The experimental configuration generally consists in a ring cavity containing a polarizing isolator placed between two polarization controllers. The basic principle is the following. The polarization state evolves nonlinearly during the propagation of the pulse in the fiber due to the combined effects of self-phase modulation and cross-phase modulation induced on the two orthogonal polarization components, both resulting from the optical Kerr effect. A polarization controller is adjusted at the output of the fiber such that the polarizing isolator lets pass the central intense part of the pulse but blocks the low-intensity pulse wings. This technique of nonlinear polarization rotation for passive mode locking leads to stable, self-starting pulse trains. Theoretical approaches to this problem can be divided into two categories. The first one has been proposed by Haus et al [7] and consists in writing a phenomenological scalar equation (the master mode-locking equation) assuming that all effects per pass are small. The model includes the group velocity dispersion (GVD), the optical Kerr nonlinearity and a gain medium. No birefringence is taken into account. Although the mode-locking properties of the laser can be described through this approach for positive and negative GVD, the stability of the mode-locked solutions as a function of the orientation of the polarization controllers cannot be described in this way. On the other hand Kim et al [8] use an approach based on two coupled nonlinear Schrödinger equations. The medium is assumed to be birefringent. The GVD together with the Kerr effect are taken into account. The orientation angles of the eigenaxis of the fiber at both sides of the polarizer are explicitly taken into account through a periodic perturbation. Numerical simulations show that mode-locking can be achieved for negative GVD. To our best knowledge, none of the models take into account all the characteristics of the fiber (birefringence, GVD, gain, optical Kerr effect) and the action of the orientation of the polarization controllers.

The aim of this paper is to investigate experimentally and theoretically the mode-locking properties of the Yb-doped double-clad fiber laser. Modelocking is achieved through nonlinear polarization rotation in a unidirectional ring cavity containing a polarizer placed between two half-wave plates. This paper is organized as follows. Section II is devoted to the experimental re- 
sults. We first briefly recall the operating regime of the laser as a function of the orientation of the two half-wave plates. A stability diagram of the mode-locked laser is presented [9]. The parameters required in the modelling section are measured. The birefringence of the double-clad fiber is first determined using the magneto-optic method [10]. The total dispersion has been estimated from other experiments where compression of the pulses was realized [11, 12]. In section 3 the stability of the mode-locking regime is theoretically investigated. The analysis is based on the coupling between the two linearly polarized eigenstates of the fiber [8] [13]. The propagation problem is modelled through two nonlinear Schrodinger equations in a gain medium where the GVD, the nonlinear coefficient and the gain are assumed small over the cavity length. The eigenaxis of the fiber at each side of the polarizer are referenced with respect to the polarizing axis. These angles can be adjusted with two half-wave plates and will thus be considered as variable in the analysis. The polarizer is treated as a periodic perturbation acting as a projecting operator. Using the expressions derived in the propagation problem, we calculate the electric field amplitude just after the polarizer as a function of the corresponding amplitude at the previous round-trip. The round-trip number is then considered as a continuous variable, which allows us to use a multiscale analysis approach. The final equation is a complex Ginzburg-Landau equation whose coefficients explicitly involve the orientation angles of the half-wave plates. Explicit solutions are given and their stabilities are discussed. A stability diagram of the mode-locked regime is calculated and compared to the experimental diagram.

\section{Experimental results}

In this section we briefly recall the results obtained with an Yb-doped doubleclad fiber laser in a unidirectional ring cavity operating at $1.08 \mu \mathrm{m}$ [9]. A polarizing isolator is placed between two half-wave plates. Although this configuration is known to generate ultrashort pulses in low power fiber lasers, it can also lead to very different regimes depending on the orientation of the polarization elements, such as cw operation, Q-switching, mode-locking, or unstable emission regime. We thus perform a systematic study of the operating regime as a function of the relative orientation of the two halfwave plates. The experimental setup is shown in figure 11. The 4 meter long Yb-doped double-clad fiber is side-pumped using the v-groove technique [3]. 


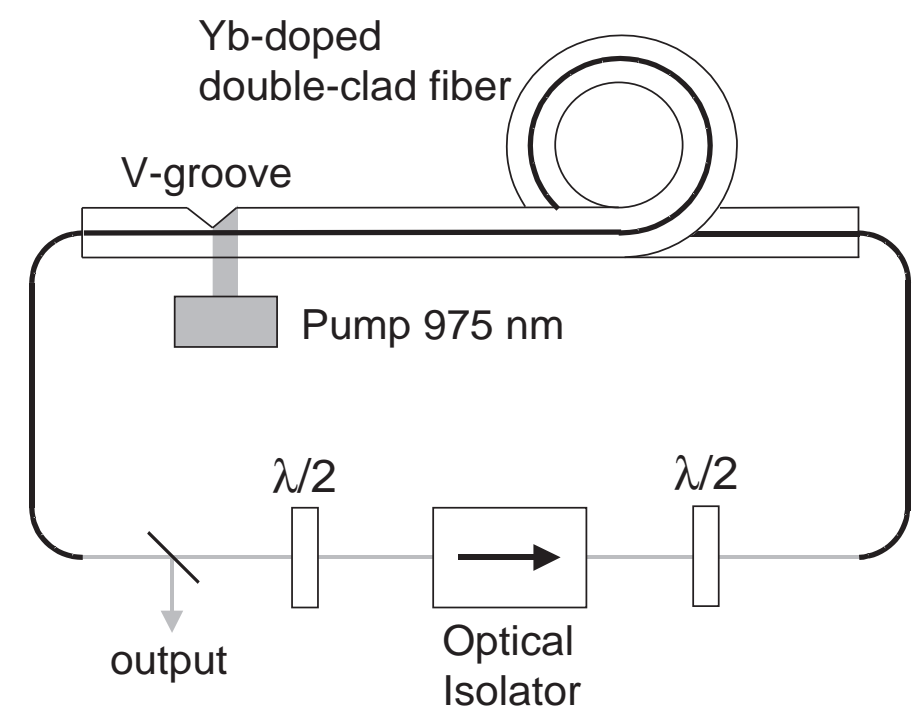

Figure 1: Experimental setup.

The launching efficiency is about 70 percent. This pumping scheme is very convenient for ring laser applications because the two fiber ends remain free. The fiber core diameter is $7 \mu \mathrm{m}$ allowing a single-mode laser signal propagation. The inner cladding is a $125 \times 125 \mu \mathrm{m}^{2}$ square leading to a multimode pump propagation. The geometry of the inner cladding ensures an efficient pump absorption. Indeed, the pump power is almost completely absorbed along the doped fiber. In addition, multimode propagation is required for high injection efficiency of large area laser diodes. Two single-mode fibers at $1 \mu \mathrm{m}$ are spliced at both ends of the double-clad fiber leading to a total fiber length of about $8.5 \mathrm{~m}$, the cavity length is about $9 \mathrm{~m}$. Fiber ends are angle-cleaved in order to avoid any Fresnel reflection which could generate a standing-wave operating regime. The splicing losses are less than $0.1 \mathrm{~dB}$. The doped fiber is pumped at $975 \mathrm{~nm}$ with a $4 \mathrm{~W}$ semiconductor laser. A bulk polarizing optical isolator is used to obtain a travelling wave laser. The latter is placed between two half-wave plates at $1.08 \mu \mathrm{m}$. Each plate can be rotated in a plane perpendicular to the propagation direction. A partially reflecting mirror ensures an output coupling of about 90 percent. The output beam is analyzed through different means. A fast photodiode is used when the laser is cw, Q-switched or unstable. In the regular mode-locking regime, 
we use an optical autocorrelator to measure the duration of the pulses. Note that there is no compression system in the cavity. An optical spectrum analyzer (Advantest) together with a microwave spectrum analyzer (Tektronix) are also used when needed. Finally, a powermeter allows us to measure the average output power.

We investigate now the operating regime of the laser as a function of the orientation of the two waveplates. $\theta_{1}\left(\theta_{2}\right)$ is the orientation of one eigenaxis of the first (second) half-wave plate referenced to the input (output) polarizer of the optical isolator. The angle between the input and output polarizer of the isolator is 45 degrees. For a given pumping power we record the evolution of the output intensity versus $\theta_{1}$ and $\theta_{2}$. Practically, we fix $\theta_{1}$ and measure the particular values of $\theta_{2}$ for which a change in the operating regime occurs. It is convenient to represent these results in the plane $\left(\theta_{1}, \theta_{2}\right)$. It allows to easily identify the output regime of the laser. The experimental results, obtained for a pumping power of about $2.75 \mathrm{~W}$, are summarized in figure 2. We have experimentally observed that the different output regimes are

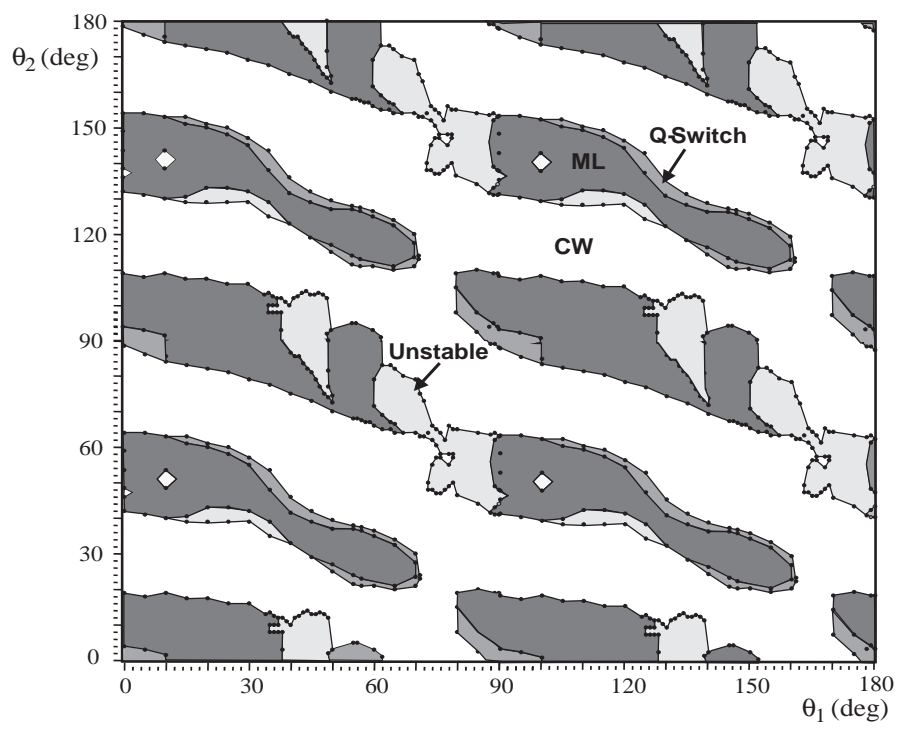

Figure 2: Operating regime of the laser as a function of the orientation of the two halfwave plates $\theta_{1}$ and $\theta_{2}$. The pump power was $2.75 \mathrm{~W}$. ML stands for mode-locked regime and $\mathrm{cw}$ for continuous wave regime.

periodic versus $\theta_{1}$ and $\theta_{2}$ with a period of 90 degrees. Figure 2 shows that the laser essentially operates in $\mathrm{cw}$, mode-lock or unstable regime while the 
Q-switch behavior appears near the boundary between cw and mode-lock regimes. Note that since the operating regime depends on the pumping rate, the mapping of figure 1 is pump power dependent. Typically, when the pump power decreases (increases), the ranges of mode-locking decrease (increase).

The different dynamical behaviors observed in our experiment have been discussed in details in reference [9]. Here, we concentrate our attention on the mode-locked operating regime. In figure 3 we give the results obtained for $\theta_{1}=20 \mathrm{deg}$ and $\theta_{2}=0 \mathrm{deg}$. In this case, the laser is first cw just above threshold (figure 3a). While the pump power is increased, an irregular self-pulsing regime appears which finally becomes a regular self-mode-locking regime. As previously discussed in the introduction, the basic principle responsible for the mode-locking is well known in such configurations. It is based on Kerr effect through nonlinear polarization rotation. This technique is also called Polarization-Additive Pulse Mode-locking. The repetition rate of the pulses corresponds to the free spectral range of the cavity, about $20 \mathrm{MHz}$ in our case. Auto-correlation traces showed a coherence spike of about $150 \mathrm{fs}$ on a longer pedestal of about 60 ps [6]. A typical spectrum in the mode-locking range is given in figure $3 \mathrm{~b}$. The mode-locked spectrum bandwidth is more than $25 \mathrm{~nm}$. This spectrum suggests that shorter pulses can be achieved by a suitable dispersion compensation. We have recently shown that femtosecond pulses are obtained with a grating pair inserted in the cavity [6]. In the present mode-locked regime, we measure $600 \mathrm{~mW}$ output power for $3.25 \mathrm{~W}$ pump power, which corresponds to high energy pulses of about $30 \mathrm{~nJ}$.

Consider now the measurements of parameters required in the theoretical section. We first measure the beating length $L_{B}$ of the double-clad fiber using the magneto-optic method [10]. The method consists to apply a sinusoidal magnetic field produced by a coil in a small portion of the fiber and to move the coil along the fiber axis. The fiber is placed between an analyzer and a polarizer. This system is then illuminated and analyzed in transmission. The detected output power is a periodic signal versus the coil displacement. The period in the spatial domain coincides with the beating length of the fiber. We found $L_{B}=50 \mathrm{~cm}$ leading to a birefringence parameter $K=1 /\left(L_{B}\right)=$ $2 \mathrm{~m}^{-1}$. We must also consider the birefringence of the undoped fibers. It has been measured to be of about $1 \mathrm{~m}^{-1}$. In the following we will consider an average value of the birefringence of about $1.5 \mathrm{~m}^{-1}$. A second important parameter is the GVD. It has been estimated from compression experiments [12. We find $\beta_{2}=0.026 \mathrm{ps}^{2} / \mathrm{m}$. 

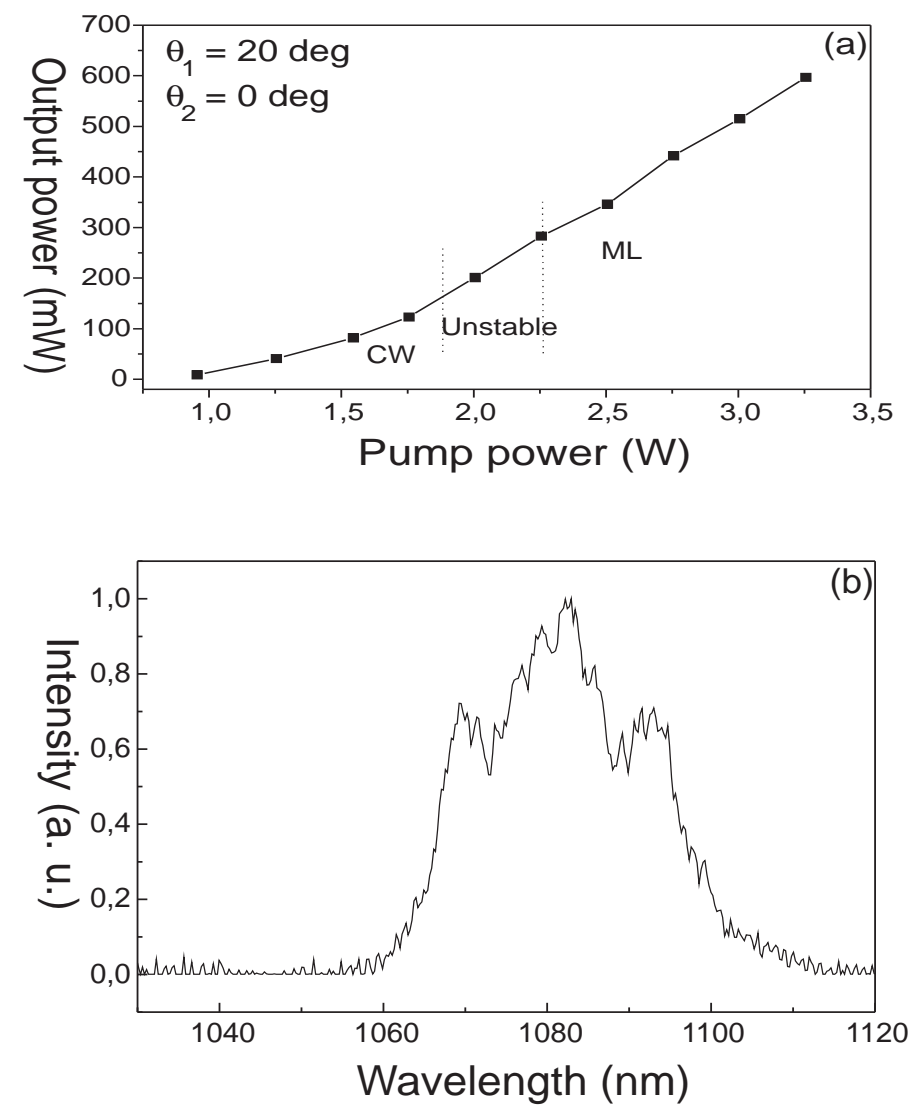

Figure 3: $\theta_{1}=20 \mathrm{deg}$ and $\theta_{2}=0 \mathrm{deg}$. (a) laser characteristic and (b) typical optical spectrum in the mode-locked regime.

\section{Theory}

The aim of this section is to develop a theoretical model able to describe the mode-locking properties of the Yb-doped fiber laser as a function of the orientation of the two half-wave plates. For that, we consider a birefringent fiber in a unidirectional ring cavity with an intracavity polarizer. The orientation of the eigenaxis of the fiber at each sides of the polarizer are unspecified and will be taken as variable parameters. This is equivalent to considering that the eigenaxis of the fiber are fixed and two half-wave plates are used to adjust their orientations with respect to the polarizer. 


\subsection{Derivation of a master equation}

\subsubsection{Propagation in the fiber}

The starting point are the equations giving the evolution of the two polarization components in a gain medium with Kerr nonlinearity and GVD. In the framework of the eigenaxis of the birefringent fiber moving at the group velocity, the pulse envelope evolution is described by the following system [8] 13]:

$$
\begin{aligned}
& i \partial_{z} u-K u-\frac{\beta_{2}}{2} \partial_{t}^{2} u+\gamma\left(u|u|^{2}+A u|v|^{2}+B v^{2} u^{*}\right)=i g\left(1+\frac{1}{\omega_{g}^{2}} \partial_{t}^{2}\right) u \\
& i \partial_{z} v+K v-\frac{\beta_{2}}{2} \partial_{t}^{2} v+\gamma\left(v|v|^{2}+A v|u|^{2}+B u^{2} v^{*}\right)=i g\left(1+\frac{1}{\omega_{g}^{2}} \partial_{t}^{2}\right) v
\end{aligned}
$$

where $\partial_{t}$ denotes the partial derivative operator $\frac{\partial}{\partial t}$. $\beta_{2}\left(\right.$ in $\left.\mathrm{ps}^{2} / \mathrm{m}\right)$ is the GVD coefficient. $K\left(\right.$ in $\left.\mathrm{m}^{-1}\right)$ is the birefringent parameter and is related to the $x$ and $y$ refractive indexes through the relation $K=\pi\left(n_{x}-n_{y}\right) / \lambda$, where lambda is the optical wavelength. $\gamma=2 \pi n_{2} /\left(\lambda A_{e f f}\right)$ is the nonlinear coefficient, $n_{2}$ (in $\left.\mathrm{m}^{2} / \mathrm{W}\right)$ is the nonlinear index coefficient and $A_{\text {eff }}\left(\right.$ in $\mathrm{m}^{2}$ ) the effective core area of the fiber. $A$ and $B$ are the dielectric coefficients. In isotropic media, $A=2 / 3$ and $B=1 / 3$ [13]. This is the case with our silicate fibers. $g$ is the gain coefficient $\left(\right.$ in $^{-1}$ ) and $\omega_{g}$ is the spectral gain bandwidth (in $\mathrm{ps}^{-1}$ ). At first order the gain coefficient is fixed by the fact that it compensates the losses. Note that polarization mode dispersion is not taken into account since the cavity length is short (about $9 \mathrm{~m}$ ). For numerical simulations we will use the following values for the parameters: $K=1.5 \mathrm{~m}^{-1}, \beta_{2}=0.026 \mathrm{ps}^{2} / \mathrm{m}, L=9 \mathrm{~m}, \gamma=3 \cdot 10^{-3} \mathrm{~W}^{-1} \mathrm{~m}^{-1}$ and $\omega_{g}=10^{13} \mathrm{~s}^{-1}$.

We now assume that the GVD $\beta_{2}$, the nonlinear coefficient $\gamma$, the gain filtering $\rho=g / \omega_{g}^{2}$ are small over one round-trip of the cavity. We introduce a small parameter $\varepsilon$ and replace these quantities by $\varepsilon \beta_{2}, \varepsilon \gamma$ and $\varepsilon \rho$. We then look for solutions of the system (1-2) under the form of a power series in $\varepsilon$, as

$$
\begin{aligned}
& u=u_{0}+\varepsilon u_{1}+O\left(\varepsilon^{2}\right), \\
& v=v_{0}+\varepsilon v_{1}+O\left(\varepsilon^{2}\right) .
\end{aligned}
$$

At order $\varepsilon^{0}$ it yields

$$
u_{0}=\tilde{u}_{0} e^{(g-i K) z}
$$




$$
v_{0}=\tilde{v}_{0} e^{(g+i K) z},
$$

where $\tilde{u}_{0}$ and $\tilde{v}_{0}$ do not depend on $z$, ie depend on the time variable $t$ only.

Making the transformation

$$
\begin{aligned}
& u_{1}=\tilde{u}_{1}(z, t) e^{(g-i K) z}, \\
& v_{1}=\tilde{v}_{1}(z, t) e^{(g+i K) z},
\end{aligned}
$$

the equations obtained at order $\varepsilon$ can be integrated with regard to $z$ to yield, for the component $u$ :

$$
\begin{aligned}
\tilde{u}_{1}= & \tilde{u}_{1}(0)+z\left(\rho-\frac{i \beta_{2}}{2}\right) \partial_{t}^{2} \tilde{u}_{0} \\
& +i \gamma\left(\tilde{u}_{0}\left|\tilde{u}_{0}\right|^{2}+A \tilde{u}_{0}\left|\tilde{v}_{0}\right|^{2}\right) \frac{e^{2 g z}-1}{2 g}+i \gamma B \tilde{v}_{0}^{2} \tilde{u}_{0}^{*} \frac{e^{(2 g+4 i K) z}-1}{2 g+4 i K} .
\end{aligned}
$$

To get the complete expression for $u(z)$, we notice that

$$
u(0)=\tilde{u}_{0}+\varepsilon \tilde{u}_{1}(0)+O\left(\varepsilon^{2}\right) .
$$

Then the two components of the wave amplitude can be written as

$$
\begin{aligned}
u(z)= & u(0) e^{(g-i K) z}+\varepsilon\left[z\left(\rho-\frac{i \beta_{2}}{2}\right) \partial_{t}^{2} u(0)\right. \\
& +i \gamma\left(u(0)|u(0)|^{2}+A u(0)|v(0)|^{2}\right) \frac{e^{2 g z}-1}{2 g} \\
& \left.+i \gamma B \tilde{v}(0)^{2} \tilde{u}(0)^{*} \frac{e^{(2 g+4 i K) z}-1}{2 g+4 i K}\right] e^{(g-i K) z}+O\left(\varepsilon^{2}\right), \\
v(z)= & v(0) e^{(g+i K) z}+\varepsilon\left[z\left(\rho-\frac{i \beta_{2}}{2}\right) \partial_{t}^{2} v(0)\right. \\
& +i \gamma\left(v(0)|v(0)|^{2}+A v(0)|u(0)|^{2}\right) \frac{e^{2 g z}-1}{2 g} \\
& \left.+i \gamma B \tilde{u}(0)^{2} \tilde{v}(0)^{*} \frac{e^{(2 g-4 i K) z}-1}{2 g-4 i K}\right] e^{(g+i K) z}+O\left(\varepsilon^{2}\right) .
\end{aligned}
$$

\subsubsection{Polarizer}

Figure 4 shows the respective orientation of the eigenaxis of the fiber before and after the polarizer (optical isolator). $\left(u_{-}, v_{-}\right)$are the field components 


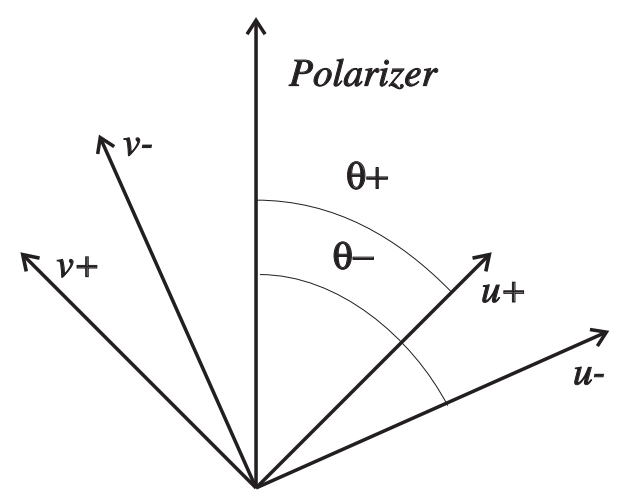

Figure 4: Definition of the angles $\theta_{-}$and $\theta_{+}$.

just before the polarizer, $\left(u_{+}, v_{+}\right)$just after it. The effect of the polarizer can be written as

$$
\left(\begin{array}{l}
u_{+} \\
v_{+}
\end{array}\right)=\beta\left(\begin{array}{c}
\cos \theta_{+} \\
\sin \theta_{+}
\end{array}\right)\left(\begin{array}{ll}
\cos \theta_{-} & \sin \theta_{-}
\end{array}\right)\left(\begin{array}{l}
u_{-} \\
v_{-}
\end{array}\right),
$$

where $\beta$ is its transmission coefficient $(\beta<1)$. Immediately after the polarizer, the field has a well-defined linear polarization. Denote by $f_{n}$ its amplitude at the beginning of the $n^{\text {th }}$ round trip. Equation (12) can be written as

$$
f_{n+1}=\beta\left(\begin{array}{ll}
\cos \theta_{-} & \sin \theta_{-}
\end{array}\right)\left(\begin{array}{c}
u_{-, n} \\
v_{-, n}
\end{array}\right),
$$

and

$$
\left(\begin{array}{c}
u_{+, n+1} \\
v_{+, n+1}
\end{array}\right)=\left(\begin{array}{c}
\cos \theta_{+} \\
\sin \theta_{+}
\end{array}\right) f_{n+1}
$$

where $\left(u_{n}, v_{n}\right)$ are the field components during the $n^{\text {th }}$ round. The field components $u_{+n}=u_{n}(0)$ and $v_{+n}=v_{n}(0)$, at the entrance of the fiber immediately after the polarizer, are transformed respectively into $u_{n}(L)=$ $u_{-n}$ and $v_{n}(L)=v_{-n}$, at the end of the fiber immediately before the polarizer ( $L$ is the fiber length).

$u_{n}(0)$ and $v_{n}(0)$ are proportional to $f_{n}$. Using the propagation formulas (11) and (12), $u_{n}(L)$ and $v_{n}(L)$ can be computed. Then $f_{n+1}$ is computed as a function of $f_{n}$ using (14). This yields

$$
f_{n+1}=\beta e^{g L}\left\{Q f_{n}+\varepsilon\left[\left(\rho-\frac{i \beta_{2}}{2}\right) L Q \partial_{t}^{2} f_{n}+i P f_{n}\left|f_{n}\right|^{2}\right]\right\}+O\left(\varepsilon^{2}\right),
$$


with

$$
Q=\cos \left(\theta_{+}-\theta_{-}\right) \cos K L-i \cos \left(\theta_{+}+\theta_{-}\right) \sin K L
$$

and

$$
\begin{aligned}
& P=\gamma\left(\frac { e ^ { 2 g L } - 1 } { 2 g } \left[\cos \left(\theta_{+}-\theta_{-}\right) \cos K L-i \cos \left(\theta_{+}+\theta_{-}\right) \sin K L\right.\right. \\
& \left.+\frac{A-1}{2} \sin 2 \theta_{+}\left[\sin \left(\theta_{+}+\theta_{-}\right) \cos K L-i \sin \left(\theta_{+}-\theta_{-}\right) \sin K L\right]\right] \\
& +\frac{B}{2} \sin 2 \theta_{+}\left[\sin \theta_{+} \cos \theta_{-} e^{-i K L} \frac{e^{(2 g+4 i K) L}-1}{2 g+4 i K}\right. \\
& \left.\left.+\cos \theta_{+} \sin \theta_{-} e^{i K L} \frac{e^{(2 g-4 i K) L}-1}{2 g-4 i K}\right]\right) .
\end{aligned}
$$

\subsubsection{Gain threshold and continuous limit for $f_{n}$}

The dominant behavior of $\left(f_{n}\right)$ is given by the zero order term in (16):

$$
f_{n+1}=\beta e^{g L} Q f_{n}+O(\varepsilon) .
$$

A steady state over a large number of round trips could be reached if this first order evolution is the multiplication by a phase factor, but only in this case. Therefore the modulus of $\beta e^{g L} Q$ must be 1 . This gives the threshold gain value:

$$
\begin{aligned}
g_{0} & =\frac{-1}{2 L} \ln \left(\beta^{2}|Q|^{2}\right) \\
& =\frac{-1}{2 L} \ln \left(\beta^{2}\left[\cos ^{2}\left(\theta_{+}-\theta_{-}\right)-\sin 2 \theta_{+} \sin 2 \theta_{-} \sin ^{2} K L\right]\right) .
\end{aligned}
$$

Denote by $e^{i \psi}$ the quantity $\beta e^{g_{0} L} Q$. The exact condition is $\left|\beta e^{g L} Q\right|=1+$ $O(\varepsilon)$, the gain $g$ therefore writes $g=g_{0}+\varepsilon g_{1}$, in which $g_{1}$ is still free. Expanding $e^{\varepsilon g_{1} L}$ in a power series of $\varepsilon$, equation (16) becomes

$$
f_{n+1}=e^{i \psi}\left(1+\varepsilon g_{1} L\right) f_{n}+\varepsilon\left(\rho-\frac{i \beta_{2}}{2}\right) L e^{i \psi} \partial_{t}^{2} f_{n}+i \varepsilon \frac{P e^{i \psi}}{Q} f_{n}\left|f_{n}\right|^{2}+O\left(\varepsilon^{2}\right) \text {. }
$$

Consider now some function $f$ of a continuous variable $z$, obeying an equation of the form

$$
i \partial_{z} f=(\mathcal{A}+i \varepsilon \mathcal{B}) f+\varepsilon \mathcal{C} \partial_{t}^{2} f+\varepsilon \mathcal{D} f|f|^{2},
$$


where $\mathcal{A}$ and $\mathcal{B}$ are real, and $\mathcal{C}$ and $\mathcal{D}$ complex coefficients. We look for solutions under the form $f=f_{0}+\varepsilon f_{1}+\ldots$. The solution at first order is

$$
f_{0}=\tilde{f}_{0} e^{-i \mathcal{A} z}
$$

We write

$$
f_{1}=\tilde{f}_{1}(z, t) e^{-i \mathcal{A} z}
$$

and obtain:

$$
f(L)=\left[f(0)+\varepsilon\left(\mathcal{B} f(0)-i \mathcal{C} \partial_{t}^{2} f(0)-i \mathcal{D} f(0)|f(0)|^{2}\right) L\right] e^{-i \mathcal{A} L}+O\left(\varepsilon^{2}\right) .
$$

Equations (21) and (25) are identified according to $f(0) \equiv f_{n}$ and $f(L) \equiv$ $f_{n+1}$, to yield

$$
\begin{gathered}
e^{-i \mathcal{A} L}=e^{i \psi}, \\
\mathcal{B}=g_{1}, \\
\mathcal{C}=\frac{\beta_{2}}{2}+i \rho, \\
\mathcal{D}=\frac{-P}{Q L} .
\end{gathered}
$$

Then the continuous equation (22) yields some interpolation of the discrete sequence $\left(f_{n}\right)$. The continuous approximation is relevant when the number of round trips is very large; further, mode-locked pulses actually correspond to this situation. The small correction of order $\varepsilon$ in equations (22) or (25) gives account for the variations of $f$ on 'propagation distances', which are here number of round trips, very large, of order $1 / \varepsilon$. This can be shown in a rigorous way using the multiscale formalism, commonly used for the derivation of the model equations in the soliton theory [15]. We introduce a slow variable $\zeta=\varepsilon z$, in such a way that

$$
\partial_{z}=\partial_{\hat{z}}+\varepsilon \partial_{\zeta}
$$

The values of $\zeta$ about 1 correspond to number of round trips about $1 / \varepsilon$. $f$ is expanded as above as $f_{0}+\varepsilon f_{1}+\ldots$, and the first order obviously yields (23), we make the transform (24) and $\tilde{f}_{1}$ must satisfy the following equation:

$$
i \partial_{\zeta} \tilde{f}_{0}+i \partial_{\hat{z}} \tilde{f}_{1}=i \mathcal{B} \tilde{f}_{0}+\mathcal{C} \partial_{t}^{2} \tilde{f}_{0}+\mathcal{D} \tilde{f}_{0}\left|\tilde{f}_{0}\right|^{2}
$$


Equation (31) can be written as

$$
\partial_{\hat{z}} \tilde{f}_{1}=\mathcal{F}\left(\tilde{f}_{0}\right) .
$$

The long distance evolution of $\tilde{f}_{0}$ is obtained from the requirement that $\tilde{f}_{1}$ does not grow linearly with $z$. This yields $\partial_{\hat{z}} \tilde{f}_{1}=0$ and

$$
i \partial_{\zeta} \tilde{f}_{0}=i \mathcal{B} \tilde{f}_{0}+\mathcal{C} \partial_{t}^{2} \tilde{f}_{0}+\mathcal{D} \tilde{f}_{0}\left|\tilde{f}_{0}\right|^{2}
$$

Or, using real coefficients only:

$$
i \partial_{\zeta} \tilde{f}_{0}=i g_{1} \tilde{f}_{0}+\left(\frac{\beta_{2}}{2}+i \rho\right) \partial_{t}^{2} \tilde{f}_{0}+\left(\mathcal{D}+i \mathcal{D}_{i}\right) \tilde{f}_{0}\left|\tilde{f}_{0}\right|^{2}
$$

Equation (33) or (34) is the cubic complex Ginzburg-Landau (CGL) equation. Note that equation (33) is formally identical to the master equation proposed by Haus et al [7]. However, the coefficients of (33) explicitly depend on the orientation of the eigenaxis of the fiber at both sides of the polarizer. An essential feature is the arising of a nonlinear gain or absorption $\mathcal{D}_{i}$, which results from the combined effects of the nonlinear rotation of the polarization, the losses due to the polarizer, and the linear gain. The value and the sign of $\mathcal{D}_{i}$ depend on the angles $\theta_{+}$and $\theta_{-}$between the eigenaxis of the fiber and the polarizer.

\subsection{Different regimes for the CGL equation}

\subsubsection{The stationary solution and its modulational instability}

The CGL equation (34) admits the following nonzero stationary solutions, ie with a constant modulus:

$$
f_{1}=A e^{i(\kappa \zeta-\Omega t)}
$$

with

$$
\Omega^{2}=\frac{1}{\rho}\left(\mathcal{D}_{i}|A|^{2}+g_{1}\right)
$$

and

$$
\kappa=\frac{\beta_{2}}{2 \rho}\left(\mathcal{D}_{i}|A|^{2}+g_{1}\right)-\mathcal{D}_{r}|A|^{2} .
$$

In the particular case $\Omega=0$ it is a constant solution (independent of $t$ ), with a fixed amplitude

$$
A=\sqrt{\frac{-g_{1}}{\mathcal{D}_{i}}}
$$


and

$$
\kappa=\frac{\mathcal{D}_{r}}{\mathcal{D}_{i}} g_{1}
$$

It exists only if $\mathcal{D}_{i} g_{1}<0$, ie if the excess of linear gain $g_{1}$ and the effective nonlinear gain $\mathcal{D}_{i}$ have opposite signs. We perform a linear stability analysis for the constant solution $f_{c}=A e^{i \kappa \zeta}$. We seek for solutions of the form $f_{1}=f_{c}(1+u), u$ being very small. It is seen that it must satisfy the following equation:

$$
-\kappa u+i \partial_{\zeta} u=i g_{1} u+\left(\frac{\beta_{2}}{2}+i \rho\right) \partial_{t}^{2} u+\mathcal{D} A^{2}\left(2 u+u^{*}\right) .
$$

Due to the term $u^{*}$, equation (40) is not linear. The eventual instability is of the type discovered first by Benjamin and Feir in the frame of water waves [16]. It has been shown that this kind of instability involves the nonlinear interaction of two modulation terms with conjugated phases with the square $A^{2}$ of the constant solution [17]. Thus we write $u$ as:

$$
u=u_{1} e^{(\lambda \zeta-i \omega t)}+u_{2} e^{\left(\lambda^{*} \zeta+i \omega t\right)}
$$

It is found that $\lambda$ satisfies an equation of the form

$$
\lambda^{2}+2 b \lambda+c=0
$$

Using the expression (38) of the fixed amplitude $A$, we get the following values of the real constants $b$ and $c$ :

$$
\begin{gathered}
b=g_{1}+\rho \omega^{2}, \\
c=\left(g_{1} \frac{\mathcal{D}_{r}}{\mathcal{D}_{i}}+\frac{\beta_{2}}{2} \omega^{2}\right)^{2}+b^{2}-g_{1}^{2}\left(1+\frac{\mathcal{D}_{r}^{2}}{\mathcal{D}_{i}^{2}}\right) .
\end{gathered}
$$

A short analysis of equation (42) show that a necessary condition for $u$ to remain bounded is that $b$ is positive. This proves that modulational instability occurs as soon as the excess of linear gain $g_{1}$ is negative and the nonlinear gain $\mathcal{D}_{i}$ is positive. Recall indeed that the existence of the constant nonzero solution requires that these two quantities have opposite signs. When $g_{1}>0$ and $\mathcal{D}_{i}<0$, we show that the modulational instability never occurs. Numerical computation shows indeed that the quantity $\left(\mathcal{D}_{r} \beta_{2} / 2+\mathcal{D}_{i} \rho\right)$ is always negative, which ensures, using elementary analysis, that $c$ is always positive, and the result follows. 
If we admit that the excess of linear gain $g_{1}$ will self-adjust to a value for which a stable solution exists, the above discussion shows that the stability of the constant solution depends on the sign of the effective nonlinear gain $\mathcal{D}_{i}$. It is stable when $\mathcal{D}_{i}<0$ only. We expect to observe continuous laser emission when the constant solution is stable, and only in this case. Thus the domains of continuous emission should coincide with the regions where $\mathcal{D}_{i}$ is negative. The sign of $\mathcal{D}_{i}$ as function of the angles $\left(\frac{-1}{2} \theta_{-}, \frac{1}{2} \theta_{+}\right)$is drawn on figure 5. It is easily checked that the relation between the angles $\theta_{1}, \theta_{2}$ used in the experiments and the angles $\theta_{+}, \theta_{-}$defined by figure 1 is such that $\theta_{1}=\frac{-1}{2} \theta_{-}+\theta_{10}$ and $\theta_{2}=\frac{1}{2} \theta_{+}+\theta_{20}$, with some fixed value of $\theta_{10}$ and $\theta_{20}$. The white domain on figure 5 corresponds to the negative values of

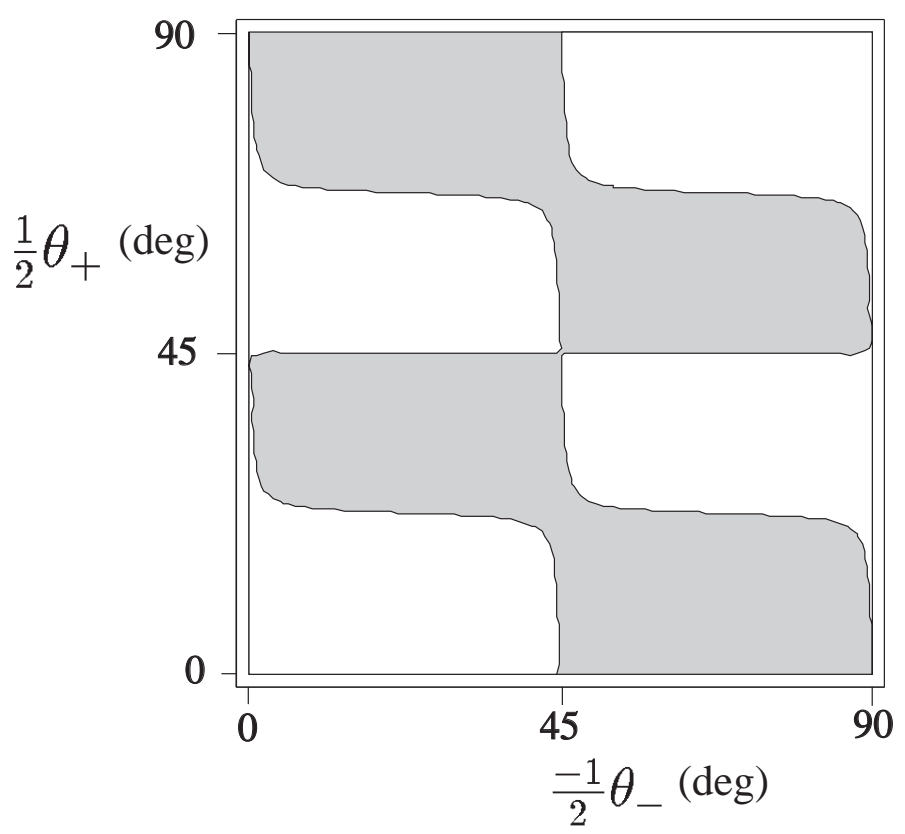

Figure 5: Sign of the effective nonlinear gain $\mathcal{D}_{i}$ as a function of the orientation of the polarizer. White: $\mathcal{D}_{i}<0$, and the constant solution of the CGL equation is stable. Gray: $\mathcal{D}_{i}>0$, and the constant solution of the CGL equation is unstable.

the nonlinear gain $\mathcal{D}_{i}$. It is thus the domain where the constant solution of the CGL equation is stable. Continuous laser emission should occur in this domain. 


\subsubsection{Localized solutions}

A localized analytical solution of the CGL equation (34) can also be written [18. It has the following expression:

$$
f_{1}=a(t) e^{i(d \ln a(t)-\omega \zeta)},
$$

with

$$
a(t)=B C \operatorname{sech}(B t)
$$

and

$$
\begin{gathered}
B=\sqrt{\frac{g_{1}}{\rho d^{2}-\rho-\beta_{2} d}}, \\
C=\sqrt{\frac{3 d\left(4 \rho^{2}+\beta_{2}^{2}\right)}{2\left(\beta_{2} \mathcal{D}_{i}-2 \rho \mathcal{D}_{r}\right)}}, \\
d=\frac{3\left(\beta_{2} \mathcal{D}_{r}+2 \rho \mathcal{D}_{i}\right)+\sqrt{9\left(\beta_{2} \mathcal{D}_{r}+2 \rho \mathcal{D}_{i}\right)^{2}+8\left(\beta_{2} \mathcal{D}_{i}-2 \rho \mathcal{D}_{r}\right)^{2}}}{2\left(\beta_{2} \mathcal{D}_{i}-2 \rho \mathcal{D}_{r}\right)}, \\
\omega=\frac{-g_{1}\left(4 \rho d+\beta_{2} d^{2}-\beta_{2}\right)}{2\left(\rho d^{2}-\rho-\beta_{2} d\right)} .
\end{gathered}
$$

The inverse $B$ of the pulse length is real only if the quantity $\mathcal{T}=\left(\rho d^{2}-\rho-\beta_{2} d\right)$ and the excess of linear gain $g_{1}$ have the same sign. The regions where $\mathcal{T}$ is either positive or negative are specified on figure 6. The background with zero amplitude is stable when the excess of linear gain $g_{1}$ is negative, and unstable in the opposite case. When $g_{1}>0$, the exact localized solution (45) is unstable due to the instability of the background [18]. Qualitatively, localized pulse formation can be expected when the excess of linear gain $g_{1}$ is negative and the nonlinear gain $\mathcal{D}_{i}$ is positive, as is suggested on figure 7 . The effective self-phase modulation $\mathcal{D}_{r}$ is always negative, and the dispersion $\beta_{2}$ is positive. Therefore their conjugated effect leads to the increase of the pulse width. If $g_{1}$ is positive and $\mathcal{D}_{i}$ negative (figure $7 \mathrm{a}$ ), the nonconservative effects decrease the amplitude at the top of the pulse, and increase it at the bottom: no stable localized pulse can be formed. If on the contrary $g_{1}$ is negative and $\mathcal{D}_{i}>0$ (figure $7 \mathrm{~b}$ ), energy appears at the top of the pulse and disappears at its bottom, yielding some pulse narrowing, which could be expected to balance the broadening caused by the nonlinear index variations. In fact the analytical localized solution (45) is never stable when $\beta_{2} \mathcal{D}_{r}<0$, 


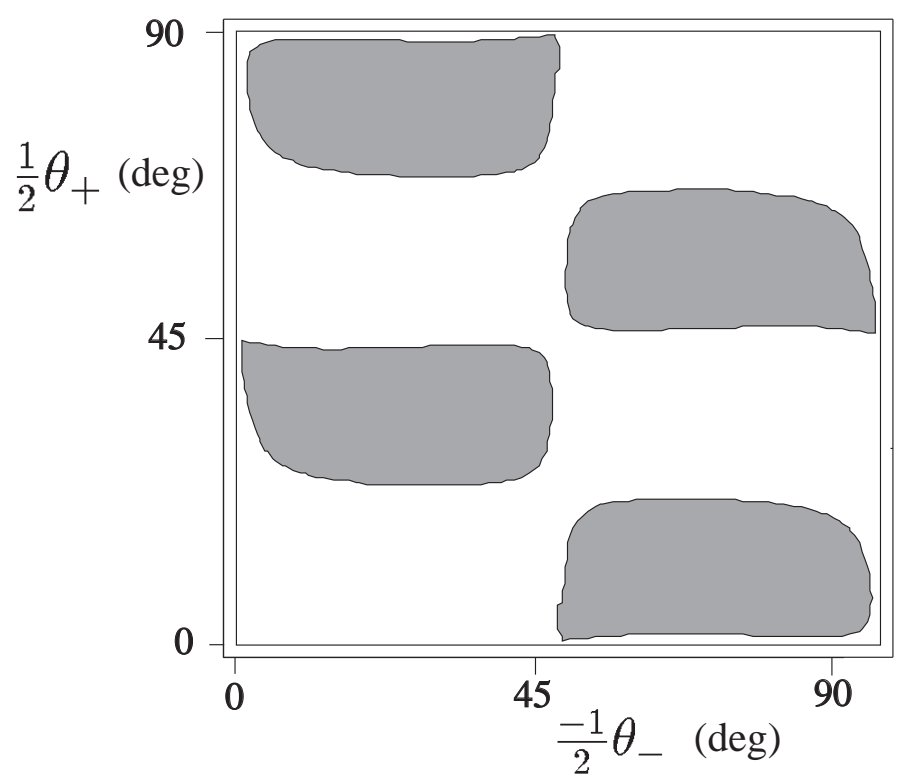

Figure 6: Sign of the excess of nonlinear gain value $g_{1}$ for which the exact localized solution exists, as a function of the orientation of the polarizer. White: $\mathcal{T}$ and $g_{1}$ positive. Gray: $\mathcal{T}$ and $g_{1}$ negative.
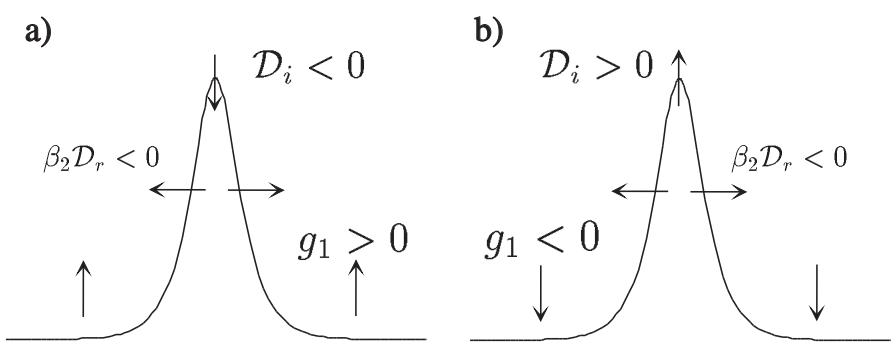

Figure 7: Schematic representation of the effect of the nonlinear gain $\mathcal{D}_{i}$, of the excess of linear gain $g_{1}$, and of both the dispersion $\beta_{2}$ and the effective self-phase modulation $\mathcal{D}_{r}$ on a localized pulse.

but can be stabilized when higher order nonlinear terms are taken into account, as shows the study of the so-called quintic CGL equation [18]. The stabilizing term should be a quintic nonlinear absorption. Observe that here the effective nonlinear gain $\mathcal{D}_{i}$ follows from the linear gain and the nonlinear evolution of the polarization in the fiber, without considering cubic nonlinear 
gain in the initial model (112). In an analogous way, an effective quintic nonlinear gain term in the master equation (33) does not require that a quintic nonlinear gain term is present in the initial model, either a cubic nonlinear gain or a quintic nonlinear index should be enough. (1-2). The precise determination of this higher order term is left for further study. On these grounds, the exact solution (45) corresponds to a potentially stable pulse when $g_{1}<0$ and $\mathcal{D}_{i}>0$, and to a completely unstable pulse when $g_{1}>0$ and $\mathcal{D}_{i}<0$.

\subsubsection{The different regimes}

According to the conclusions of the previous section, and assuming that the excess of linear gain $g_{1}$ will self-adjust to the value for which a stable solution exists, mode-locked laser emission can be expected in the region where $\mathcal{D}_{i}>0$ and $\mathcal{T}<0$. This is the domain in dark gray marked ML on figure 8 . In the region where $\mathcal{D}_{i}$ is negative, the constant solution of the CGL equation is stable, and continuous laser emission is expected to occur. It is the white domain marked $\mathrm{cw}$ on the figure 8. In the region where $\mathcal{T}$ is positive and $\mathcal{D}_{i}$ negative, no stable localized neither constant solution exist. The laser behavior is expected to be unstable in this region, in light gray on the figure 8 . The theoretical results summarized on the figure 8 can be compared to the experimental results of figure 2. A discrepancy between the two figures appears at first glance: the periodicity of the behavior regarding the variable $\theta_{2}$ or $\theta_{+} / 2$ seems to be 45 degrees according to the theory, while it is 90 degrees according to the experiment. Indeed, the two elongated domains of mode-locked behavior are not equivalent according to experimental observation, while they seem to be identical on the theoretical figure 8. A contour plot of the effective nonlinear gain $\mathcal{D}_{i}$ as a function of the angles is drawn on figure 9. It is seen that the values of $\mathcal{D}_{i}$ are not the same in the two theoretical domains of mode-locked emission. Thus they are in fact not equivalent, and the periodicity of the theoretical result is 90 degrees, as that of the experimental one. Further it has been shown [18] that an excessive value of the nonlinear gain might prevent pulse stabilization. A more accurate analysis would thus very likely show that the regions where $\mathcal{D}_{i}$ takes its highest values are out of the domain of stability of the localized pulse. This confirms that the two horizontal domains of mode-locked regime drawn on figure 8 are not equivalent. Further, it could explain a part of the discrepancy between theoretical and experimental results.

Before we conclude, let us consider the influence of the group velocity 


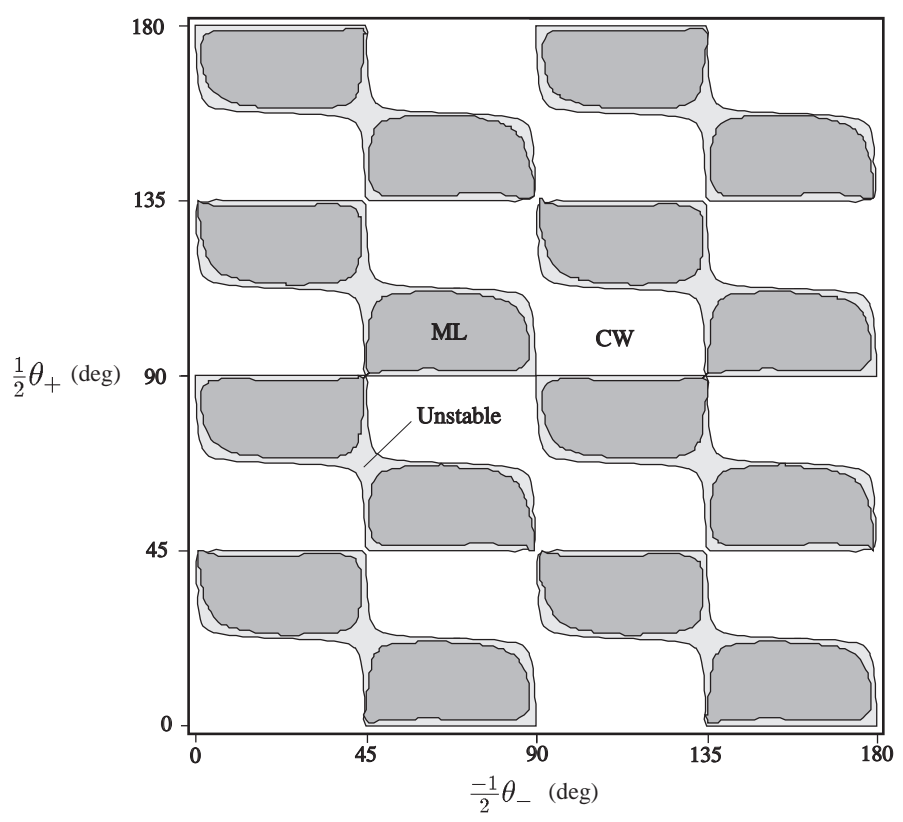

Figure 8: Operating regime of the laser as a function of the angles $\theta_{+}$and $\theta_{-}$between the polarizer and the eigenaxis of the fiber, according to the theory. ML stands for modelocked regime and $\mathrm{CW}$ for continuous wave regime.

dispersion on the stability of the mode-locking solutions. This is important because GVD compensation is required to generate subpicosecond pulses. Experimentally, we have obtain 666 fs pulses with a grating pair inserted in the cavity [6, 12]. Under these conditions, the total GVD is about $\beta_{2}=$ $0.0005 \mathrm{ps}^{2} / \mathrm{m}$. Hence, stable ultrashort pulse generation is possible for such low values of the GVD. We have tested our master equation for decreasing values of $\beta_{2}$. We have found that a lowering of $\beta_{2}$ results in a reduction of the stable mode-locking regions. In addition, mode-locking completely disappears for $\beta_{2} \lesssim 0.0015 \mathrm{ps}^{2} / \mathrm{m}$. These results are in agreement with the predictions of the Haus's model [0] but not with our experimental results. The validity of the model presented here is therefore limited to GVD values not too close to zero. Higher order terms of the GVD could be required to improve the model near the zero-dispersion point. This problem deserves further experimental and theoretical work. 




Figure 9: Contour plot of the effective nonlinear gain $\mathcal{D}_{i}$ as a function of the angles $\theta_{+}$ and $\theta_{-}$between the polarizer and the eigenaxis of the fiber.

\section{Conclusion}

We have given a rigorous derivation of the master equation, a CGL equation, that allows to discuss theoretically the different operating regimes of the passively mode-locked Yb-doped double-clad fiber laser. This equation involves a single amplitude, but gives an account of the evolution of the polarization inside the fiber through its coefficients. An effective nonlinear gain arises from the conjugated effects of the linear gain and the self-phase modulation. Its sign depends on the orientation of the polarizer. The existence and stability of the constant nonzero solution of the CGL equation has been discussed. It gives an account of the continuous emission regime of the laser. An analytical localized solution has also been given. Although it is properly speaking unstable, it allows to discuss the formation of localized pulses, ie the mode-locked regime of the laser. An unstable regime has also been identified. These theoretical results are in good accordance with the experiments. The study of higher order corrections to this model should improve these results. 


\section{References}

[1] K. Tamura, H.A. Haus and E.P. Ippen, Self-starting additive pulse mode-locking erbium fiber ring laser, Electron. Lett. 28, 2226-2228, 1992.

[2] H.A. Haus, K. Tamura, L.E. Nelson and E.P. Ippen, Stretchedpulse additive pulse mode-locking in fiber ring lasers: theory and experiment, IEEE Jour. Quant. Electron. 31, 591-598, 1995.

[3] V.J. Matsas, T.P. Newson, D.J. Richardson and D.N. Payne, Selfstarting passively mode-locked finer ring soliton laser exploiting nonlinear polarization rotation, Electron. Lett. 28, 1391-1393, 1992.

[4] V. Cautaerts, D.J. Richardson, R. Pashotta and D.C. Hanna, Stretched pulse Yb3+:silica fiber laser, Opt. Lett. 22, 316-318, 1997.

[5] R. Hofer, M. Hofer and G.A. Reider, High energy, subpicosecond pulses from a Nd-doped double-clad fiber laser, $\mathrm{Op}$ tics Com. 169, 135-139, 1999.

[6] A. Hideur, T. Chartier, M. Brunel, S. Louis, C. Özkul and F. Sanchez, Generation of high energy femtosecond pulses from a side-pumped cYb-doped double-clad fiber laser, Appl. Phys. Lett. 79, 3389 (2001).

[7] H.A. Haus, J.G. Fujimoto and E.P. Ippen, Structures for additive pulse mode locking, J. Opt. Soc. Am. B 8, 2068-2076, 1991.

[8] A.D. Kim, J.N. Kutz and D.J. Muraki, Pulse-train uniformity in optical fiber lasers passively mode-locked by nonlinear polarization rotation, IEEE Jour. Quant. Electron. 36, 465-471, 2000.

[9] A.Hideur, T.Chartier, M.Brunel, M.Salhi, C.Ozkul and F.Sanchez, Mode-lock, Q-switch and cw operation of an Ybdoped double-clad fiber ring laser, Optics Com. 198, 141-146, 2001. 
[10] T. Chartier, A. Hideur, C. Özkul, F. Sanchez, G. Stephan, Polarization properties of single-mode optical fibers with random fluctuations of birefringence, Appl. Optics. 40, 5343-5353 (2001).

[11] M.L. Dennis and I.N. Duling III, Experimental study of sideband generation in femtosecond fiber lasers, IEEE Jour. Quant. Electron. 30, 1469-1477, 1994.

[12] A. Hideur, T. Chartier, C. Özkul, M. Brunel and F. Sanchez, Experimental study of pulse compression in a side-pumped Ybdoped double-clad mode-locked fiber laser, Appl. Phys. B 74, 121-124 (2002).

[13] G.P. Agrawal, Nonlinear Fiber Optics, Academic Press, Second Edition, 1995.

[14] D.J. Ripin and L. Goldberg, High efficiency side-coupling of light into optical fibers using embedded V-grooves, Electron. Lett. 31, 2204-2205, 1995.

[15] T. Taniuti and C.-C. Wei, Reductive perturbation method in nonlinear wave propagation I. J. Phys. Soc. Japan, 24, 941-946 (1968). T. Taniuti and N. Yajima, Perturbation method for a nonlinear wave modulation. III J. Math. Phys., 14 (10), 13891397 (1973).

[16] T.B. Benjamin and J.E. Feir, The desintegration of wave trains in deep water. Part.1. Theory, J. Fluid. Mech. 27 (3), 417-430 (1967).

[17] J.T. Stuart, F.R.S. and R.C. DiPrima, The Eckhaus and Benjamin-Feir resonance mechanisms, Proc. R. Soc. Lond. A 362, 27-41 (1978).

[18] J.M. Soto-Crespo, N.N. Akhmediev, V.V. Afanasjev, and S.Wabnitz, Pulse solutions of the cubic-quintic complex Ginzburg-Landau equation in the case of normal dispersion, Phys. Rev. E 55 (4), 4783-4796 (1997). 